\title{
Twist elasticity and anchoring in a lamellar nematic phase
}

\author{
Neha M. Patel, M.R. Dodge, Min Hua Zhu, Rolfe G. Petschek, and Charles Rosenblatt ${ }^{a}$ \\ Department of Physics, Case Western Reserve University, Cleveland, \\ Ohio 44106-7079 U.S.A. \\ Marko Prehm and Carsten Tschierske \\ Martin-Luther-Universität Halle-Wittenberg, Institute of Organic \\ Chemistry, 06120 Halle, Germany
}

(August 7, 2003)

\begin{abstract}
Electrooptic measurements were performed on a lamellar nematic phase in which the mesogenic moieties lie in lamellae that are separated by partially perfluorinated side groups. The twist elastic constant $K_{22}$ and the quadratic and quartic anchoring strength coefficients are reported. $K_{22}$ is found to be considerably smaller than that of typical three-dimensional nematics, a consequence of the greatly weakened interactions between the spatially separated lamellae.
\end{abstract}

${ }^{a}$ Author for correspondence. email: rosenblatt@cwru.edu 
Self-organization of simple organic components into more complex structures is one of the most scientifically exciting and technologically promising fields of research today $[1,2]$. One dimensional structures such as fibers, two dimensional (2D) structures such as membranes, and three dimensional (3D) structures such as columnar phases may be created from simpler structural units. Very recently Tschierske, et al demonstrated that structures having the mesogenic moiety shown in Fig. 1 and with long semiperfluorinated lateral alkyl chains $R$ [e.g., $R=\left(\mathrm{CH}_{2}\right)_{6} \mathrm{C}_{10} \mathrm{H}_{21}$ ] exhibit a series of novel lamellar liquid crystalline phases, viz., lamellar isotropic (Lam- $I$ ), lamellar nematic (Lam- $N$ ), and lamellar smectic- $A$ (Lam- $A$ ) phases [2-7]. The branched compound 1 shown in Fig. 1 with $R=\mathrm{OCH}_{2} \mathrm{C}^{*} \mathrm{H}\left(\mathrm{CH}_{3}\right) \mathrm{O}\left(\mathrm{CH}_{2}\right)_{11} \mathrm{C}_{8} F_{17}$ is designed to have low transition temperatures and a broad Lam- $N$ region, with the phase sequence crystal $-112{ }^{\circ} \mathrm{C}\left(X 77{ }^{\circ} \mathrm{C}\right)-\mathrm{Lam}-A-$ $125{ }^{\circ} \mathrm{C}-$ Lam- $N-139{ }^{\circ} \mathrm{C}-$ Lam- $I-169{ }^{\circ} \mathrm{C}-3 \mathrm{D}$ isotropic, where $X$ is an undetermined mesophase. Synthesis details will be reported elsewhere [8]. Figure 2 shows a schematic representation of the three phases, which come about because the molecule contains three incompatible moieties: a rigid aromatic core, two polar diol groups that interact via cooperative hydrogen bonding, and a partially perfluorinated pendant chain $R$. The perfluorinated chain has the effect of separating the lamellae, facilitating quasi-two-dimensional versions of the three traditional liquid crystalline phases. Although a quasi-2D smectic- $A$ phase has been observed in self-assembled DNA - cationic liposome mixtures, wherein the DNA double helices lie flat between the lipid membranes $[9,10]$, such a system exhibits neither a Lam- $I$ nor Lam- $N$ phase, nor the associated phase transitions. Because of the multiple phases exhibited by $\mathbf{1}$, we now have the opportunity to examine a host of exciting scientific questions dealing with quasi-2D and 3D-to-2D crossover behavior in liquid crystals. In this paper we report on electrooptic measurements in the Lam- $N$ phase of $\mathbf{1}$ that allow us to extract the twist elastic constant $K_{22}$ associated with a distortion perpendicular to the lamellae, as well as the azimuthal anchoring properties at the two substrates. Our central result is that both of these quantities are smaller than values found in typical 3D nematics. In particular, $K_{22}$ is fully an order of magnitude smaller than bulk $3 \mathrm{D}$ values, a result associated with the 
greatly weakened interactions between lamellae.

The cell was prepared using one semi-transparent indium-tin-oxide (ITO) coated glass slide and one uncoated microscope slide. In order to facilitate the application of an in-plane electric field, a strip of ITO of width $w=375 \mu \mathrm{m}$ was etched from the first slide using a mixture of $2.5 \%$ by volume nitric acid, $47.5 \%$ hydrochloric acid, and $50 \%$ water. After cleaning, the etched slide was cemented to the hot stage of a temperature-controlled oven. Two 12.5 $\mu \mathrm{m}$ thick strips of Mylar were placed along opposite edges of the slide to serve as spacers. The oven was heated to a temperature of $150{ }^{\circ} \mathrm{C}$ and liquid crystal was placed on the slide. After the liquid crystal melted to the Lam- $I$ phase, the second slide, which was attached to a micro-translator, was pressed against the liquid crystal and Mylar spacers. The cell was secured by a metal piece fastened across the top glass slide. The oven containing the sample was placed on the rotatable stage of a polarizing microscope and observed under crossed polarizers. The sample was heated to $170{ }^{\circ} \mathrm{C}$, corresponding to the 3D isotropic phase of the liquid crystal, and subsequently was sheared at an angle of $60^{\circ}$ with respect to the axis of the etched strip by translating the top slide (attached to the micro-translator). The sample was cooled to the Lam- $I$ phase and sheared repeatedly until good alignment, with lamellae parallel to the substrates, was observed using a polarizing microscope. Thereafter, the sample was cooled to $138^{\circ} \mathrm{C}$, corresponding to the Lam- $N$ phase, and good alignment was maintained. We believe the alignment is due to a surface memory effect [11], in essence adsorption of the mesogenic moiety at the surface. In the Lam- $N$ phase the director $\widehat{n}$ of the mesogenic moiety lies in the lamellae parallel to the shear direction. A voltage $V$ was applied to the cell, causing a rotational torque on the director. If the anchoring strength were infinite, the azimuthal director orientation $\varphi(z)$ would be maximum in the center of the cell and zero at the two surfaces. Assuming the Mauguin (optical adiabatic) limit, the polarization of light entering the cell parallel to the shear direction would follow the director orientation $\varphi(z)$ through the cell, and emerge from the cell with outgoing polarization parallel to the incoming polarization [12]; no light would pass through the analyzer. For finite anchoring strength, however, the azimuthal rotation $\varphi_{s}$ at the surface is nonzero, and the incoming 
polarization splits into ordinary and extraordinary components. The intensity of light transmitted through the analyzer increases by an amount approximately proportional to $\varphi_{s}^{2}$ [13], with a rise time $\tau_{\text {on }}=\eta / 2\left(\Delta \chi E^{2}+K_{22} q^{2}\right)$ on switching on the field. Here $\eta$ is the viscosity, $\Delta \chi$ is the susceptibility anisotropy $[=\Delta \varepsilon / 4 \pi$, where $\Delta \varepsilon$ is the dielectric anisotropy], and $E=2 \mathrm{~V} / \pi \mathrm{w}$ is the electric field at the center of the gap between the electrodes [14]. Wavevector $q$ is given approximately by $\pi /(d+2 L)$, where $d \sim 12.5 \mu \mathrm{m}$ is the cell thickness and $L$ is the "extrapolation length" $K_{22} / W$. On turning off the field, the relaxation time corresponds to $\tau_{\text {off }}=\eta / 2 K_{22} q^{2}$, and the ratio $\tau_{\text {on }} / \tau_{\text {off }}=K_{22} q^{2} /\left(\Delta \chi E^{2}+K_{22} q^{2}\right)$, thereby eliminating $\eta$.

A step increase from 0 to 1.67 statv $\mathrm{cm}^{-1}\left(5 \times 10^{4} \mathrm{~V} \mathrm{~m}^{-1}\right)$ rms of a $1 \mathrm{kHz}$ sinusoidal electric field was applied to the sample, and a video that captured the response of the liquid crystal to the electric field was recorded at 20 frames per second. Assuming $L \ll d-$ this approximation later will be shown to be valid — we find $\tau_{\text {on }}=(400 \pm 50) \mathrm{ms}$. On switching off the field, $\tau_{\text {off }}$ is found to be $(800 \pm 50) \mathrm{ms}$, and thus $\tau_{\text {on }} / \tau_{\text {off }} \approx 0.5$. We now need to determine $\Delta \chi$ to extract $K_{22}$. This is problematical, as both the imperfect alignment and the existence of the side group prevent an accurate experimental determination of $\Delta \chi$. Moreover, the mesogenic unit (without the side group) forms a $3 \mathrm{D}$ smectic- $A$ phase only at very high temperatures, where conductivity becomes a problem. Thus we shall attempt to estimate $\Delta \chi$. Utilizing the GAMESS [15] generalized atomic and molecular electronic structure system program, we calculated the dipole moment in vacuum using the restricted Hartree-Fock approximation and the $631 \mathrm{G}^{*}$ basis set the for dipole moment of a low energy conformer of the mesogen with a shortened $R$ group $\left(R=\mathrm{OCH}_{3}\right.$.) We found dipole moments of 1 to 2 Debye for typical configurations that lie in the lamellar plane. Such dipole moments, given a large order parameter and the fact that the average over conformations is likely to be appreciably anisotropic, are sufficiently large that $0.08 \leq \Delta \chi \leq 0.40$, corresponding to $1 \leq$ $\Delta \varepsilon \leq 5$. It is unlikely that $\Delta \chi$ can be much larger than 0.40 - even highly polar molecules do not have much larger values of $\Delta \chi$. Nevertheless, $\Delta \chi$ might be smaller than 0.08 , 
given that hydrogen bonding and other interactions may cause large correlations between dipoles. Using an intermediate value $\Delta \chi=0.20$, corresponding to $\Delta \epsilon=2.5$, we calculate $K_{22}=(4.0 \pm 0.3) \times 10^{-8}$ dyn. The uncertainty represents experimental error, and does not account for the uncertainty in $\Delta \chi$. Using the two limiting values for $\Delta \chi$, viz., $\Delta \chi=0.08$ and $\Delta \chi=0.40$, we find $K_{22}=(1.5 \pm 0.1) \times 10^{-8}$ dyn and $(7.7 \pm 0.6) \times 10^{-8}$ dyn, respectively Although this is a rather wide range for $K_{22}$, these values are, if anything, an overestimate. Most importantly, they are considerably smaller than those found in typical 3D nematics by about an order of magnitude [12].

Further information was gleaned by measuring $\varphi_{s}$ as a function of applied voltage. At each voltage $V$, where $1.67 \leq V \leq 36.7$ statv $\mathrm{cm}^{-1}\left(5 \times 10^{4} \leq V \leq 1.1 \times 10^{6} \mathrm{~V} \mathrm{~m}^{-1}\right)$, the microscope stage was rotated by angle $\varphi_{s}$ until the image was dark; this angle corresponds to the azimuthal angle relative to the easy axes at the two surfaces $z=0$ and at $z=d$. $\varphi_{s}$ was measured as a function of $V$, and the results are shown in Fig. 3.

To extract anchoring information from Fig. 3, we need to calculate $\varphi_{s}$ as a function of field. The bulk free energy per unit area is $F_{b} \equiv \int f(z) d z$, where $f=K_{22}(d \varphi / d z)^{2} / 2-$ $\Delta \chi E^{2} \cos ^{2}(\pi / 6-\varphi) / 2$ is the free energy per unit volume for an electric field at an angle of $30^{\circ}$ with respect to $\widehat{n}$. Applying the Euler- Lagrange equation we obtain $K_{22}\left(d^{2} \varphi / d z^{2}\right)+\Delta \chi E^{2} \cos (\pi / 6-\varphi) \sin (\pi / 6-\varphi)=0$. The anchoring energy per unit area at the two surfaces is $F_{a}=\left(W_{2} \sin ^{2} \varphi+W_{4} \sin ^{4} \varphi\right) / 2$, where $W_{2}$ is the usual quadratic anchoring strength coefficient [16] and $W_{4}$ is the quartic coefficient [17]. Assuming that $d \varphi / d z=0$ in the center of the cell where $\varphi \equiv \varphi_{m}$ at $z=d / 2$, and applying torque balance at the surface where $\varphi=\varphi_{s}$ at $z=0$, we obtain after some manipulation [18]

$$
\int_{\varphi_{s}}^{\varphi_{m}}\left[\cos ^{2}\left(\pi / 6-\varphi_{m}\right)-\cos ^{2}(\pi / 6-\varphi)\right]^{-1 / 2} d \varphi=\int_{0}^{d / 2} \xi^{-1} d z
$$

where 
$\varphi_{m}=\pi / 6-\arccos \left[\left(\xi^{2}\left(W_{2} \sin \varphi_{s} \cos \varphi_{s}+2 W_{4} \sin ^{3} \varphi_{s} \cos \varphi_{s}\right)^{2} / K_{22}^{2}+\cos ^{2}\left(\pi / 6-\varphi_{s}\right)\right)^{1 / 2}\right]$

and where the electrical coherence length $\xi \equiv \sqrt{K_{22} / \Delta \chi E^{2}} . \quad \varphi_{s}$ is calculated implicitly by substituting $\varphi_{m}$ from Eq. 2 into Eq. 1.

Fixing $K_{22}=4 \times 10^{-8}$ dyn, we performed a two parameter fit of Eqs. 1 and 2 to the data in Fig. 3, finding $W_{2}=(1.3 \pm 0.2) \times 10^{-3} \mathrm{erg} \mathrm{cm}^{-2}$ and $W_{4}=(-2.5 \pm 0.4) \times 10^{-3} \mathrm{erg} \mathrm{cm}^{-2}$. The anchoring coefficient $W_{2}$ is a bit smaller than that of, but is not especially unusual for, a 3D nematic $[19,20]$. Anchoring strength coefficients measured at different laboratories and using different methodologies may differ by as much as two orders of magnitude [20]. More interesting is the large negative value of $W_{4}$ when compared to $W_{2}$. Using only the simple quadratic form for $F_{a}$, i.e., fixing $W_{4}=0$, we found that the quality of the fit is rather poor, as the calculated angle $\varphi_{s}$ saturates at too low a field. Thus, it was necessary to introduce a negative quartic coefficient, which has the effect of "softening" the anchoring at larger azimuthal rotations. A negative fourth order coefficient has been observed in the polar anchoring energy for other liquid crystals $[21,22]$. In this case its large magnitude relative to $W_{2}$ would indicate that the shear alignment may cause anchoring by a surface memory effect [11], but that this effect is quite weak for large distortions. Finally, we note that the extrapolation length $L \sim 0.3 \mu \mathrm{m}$, which indeed is much smaller than $d$. Thus our neglect of $L$ in the dynamic measurement was valid.

As a check on the foregoing results we also performed a Fréedericksz transition measurement. For this experiment, a new cell was prepared with an electrode spacing $w=500$ $\mu \mathrm{m}$, and the cell was sheared parallel to the edges of the electrodes; thus $\widehat{n} \perp \vec{E}$. A well aligned region was located midway between the electrodes, and a mask was placed over the sample so that light would pass through this region only. Light from He-Ne laser passed through a light chopper, a polarizer oriented at $22.5^{\circ}$ with respect to $\vec{E}$, a focusing lens, the sample, an analyzer, and into a detector. The detector output was fed to a lock-in amplifier referenced to the chopper frequency. A sinusoidal voltage at $713 \mathrm{~Hz}$ was applied 
to the the sample and the amplitude was ramped slowly from 0 to 1 statv $(300 \mathrm{~V})$ rms. The detector output was recorded and is shown in Fig. 4. Although noisy due to small values of $\varphi_{s}$ and the adiabatic following of the polarization, a reproducible threshold field $E_{t h}=(2.7 \pm 0.3)$ statv $\mathrm{m}^{-1}\left[(8 \pm 1) \times 10^{4} \mathrm{~V} \mathrm{~m}^{-1}\right]$ was obtained. $E_{t h}$ is given by the implicit equation $\cot \left(\pi E_{t h} / 2 E_{t h}^{0}\right)=\pi K_{22} E_{t h} /\left(W E_{t h}^{0} d\right)$, where $E_{t h}^{0}=\pi\left(K_{22} / \Delta \chi\right)^{1 / 2} / d$ is the threshold field in the limit of $W \rightarrow \infty$ [17]. Substituting values for $E_{t h}, K_{22}, \Delta \chi$, we obtain the quadratic anchoring strength coefficient $W_{2}=(1.1 \pm 0.2) \times 10^{-3} \mathrm{erg} \mathrm{cm}^{-2}$, a value consistent with that obtained from the torque measurements.

The most interesting observation is the smallness of $K_{22}$, which is about one order of magnitude smaller than typical 3D values for the twist elastic constant [12]. We would expect the splay and bend elastic constants to be at least as large as $8 k_{B} T / \pi b$, where the distance $b$ between lamellae is at least $2.7 \mathrm{~nm}[7]$ and $k_{B} T$ is the thermal energy. Such a bound would be required by the Kosterlitz-Thouless condition $[23,24]$ for non-interacting two dimensional nematics, which is unchanged by $\pi$ rotations. This inequality can be violated in a three-dimensional system only if it is weakly ordered, in which case the elastic constant is expected to be $\sim k_{B} T / \xi_{N}$ where $\xi_{N}$ is the correlation length of the nematic order parameter. But our Lam- $N$ phase is strongly ordered: The birefrigence is observed to be at most weakly temperature-dependent, even in the Lam- $A$ phase. We therefore conclude that the inter-lamellar orientational interactions are very much smaller than the intra-lamellar orientational interactions. This is analogous to what happens in some smectic- $C$ phases, particularly those that also have anticlinic phases [25]. It is reasonable to expect that the long side groups convey little information concerning the orientation of each layer from one layer to the next. It follows that the twist elastic constant $K_{22}$ results largely from the relatively long-range dispersive interactions between layers, or from the different energies associated with fluctuations in which these layers undulate. These will be treated in detail elsewhere. Nevertheless, their contributions to the elastic constants decrease with increasing lamellar separation, which is moderately large in this case. Moreover, they are a fraction of the contribution to the elastic constants even in ordinary liquid crystals [26]. 
Thus the small measured value of the twist elastic constant is quite reasonable. By tailoring the structure of the side group, in principle one can control the twist elasticity over a very large range. Clearly, this system holds promise for investigations into many quasi-2D and crossover phenomena, and the possibility of a host of other new physical phenomena and devices.

Acknowledgments: This work was supported by the National Science Foundation under grant DMR-9982020 and by the Donors of the Petroleum Research Fund, administered by the American Chemical Society, under grant 37736-AC7. 


\section{REFERENCES}

[1] J.-M. Lehn, Proc. Nat. Acad. Sci. 99, 4763 (2002).

[2] C. Tschierske, J. Mater. Chem. 11, 2647 (2001).

[3] M. Prehm, X.H. Cheng, S. Diele, M.K. Das, and C. Tschierske, J. Am. Chem. Soc. 124, $12072(2002)$.

[4] M. Kölbel, T. Beyersdorff, X.H. Cheng, C. Tschierske, J. Kain, and S. Diele, J. Am. Chem. Soc. 123, 6809 (2001).

[5] X.H. Cheng, M.K. Das, S. Diele, and C. Tschierske, Angew. Chem. 114, 4203 (2002).

[6] X.H. Cheng, M. Prehm, M.K. Das, J. Kain, U. Baumeister, S. Diele, D. Leine, A. Blume, and C. Tschierske, J. Am. Chem. Soc. 125, (2003).

[7] M. Prehm, S. Diele, M.K. Das, and C. Tschierske, J. Am. Chem. Soc. 125, 614 (2003).

[8] M. Prehm and C. Tschierske, to be published.

[9] J.O. Rädler, I. Koltover, T. Salditt, and C.R. Safinya, Science 275, 810 (1997).

[10] T. Salditt, I. Koltover, J.O. Rädler, and C.R. Safinya, Phys. Rev. Lett. 79, 2582 (1997).

[11] N.A. Clark, Phys. Rev. Lett. 55, 292 (1985).

[12] S. Chandrasekhar, Liquid Crystals, Cambridge U. Press, Cambridge (1992).

[13] Bing Wen, M.P. Mahajan, and C. Rosenblatt, Appl. Phys. Lett. 76, 1240 (2000).

[14] I.M. Syed and C. Rosenblatt, Phys. Rev. E 67, 041707 (2003).

[15] S.T. Elbert, M.S. Gordon, J.H. Jensen, S. Koseki, N. Matsunaga, K.A .Nguyen, S.J. Su, T.L. Windus, M. Dupuis, and J.A. Montgomery J.Comput.Chem. 14, 1347 (1993) .

[16] A. Rapini and M. Papoular, J. Phys. (Paris) Colloq. 30, C4-54 (1969).

[17] K.H. Yang and C. Rosenblatt, Appl. Phys. Lett. 43, 62 (1983). 
[18] P.G. DeGennes and J. Prost, The Physics of Liquid Crystals, Clarendon, Oxford (1993).

[19] B. Jérôme, Rep. Prog. Phys. 54, 391 (1991).

[20] LM. Blinov, A.Y. Kabayenkov, and A.A. Sonin, Liq. Cryst. 5, 645 (1989).

[21] H. Yokoyama and H.A. van Sprang, J. Appl. Phys. 57, 4520 (1985).

[22] Bing Wen and C. Rosenblatt, Phys. Rev. Lett. 89, 195505 (2002).

[23] M. Kosterlitz and D.J. Thouless, J. Phys. C 5, L124 (1972).

[24] J.M. Kosterlitz, J. Phys. C 7, 1046 (1974).

[25] N.M. Patel, C. Rosenblatt, and Y.-K. Yu, Phys. Rev. E 68, 011703 (2003).

[26] A. Saupe, Z. Naturforsch., 15a, 810 (1960); 15a, 815 (1960). 


\section{FIGURES}

FIG. 1. Molecule $\mathbf{1}$ showing mesogenic moiety and side group $R$.

FIG. 2. Schematic representation of lamellar phases. a) Lam- $I$ phase, b) Lam- $N$ phase, and c) Lam- $A$ phase.

FIG. 3. Azimuthal rotation $\varphi_{s}$ at the surface vs. $E^{2}$. Solid curve represents fit to the data using $K_{22}=(4.0 \pm 0.3) \times 10^{-8}$ dyn.

FIG. 4. Intensity vs. $E$ showing Fréedericksz threshold field $E_{t h}$. 


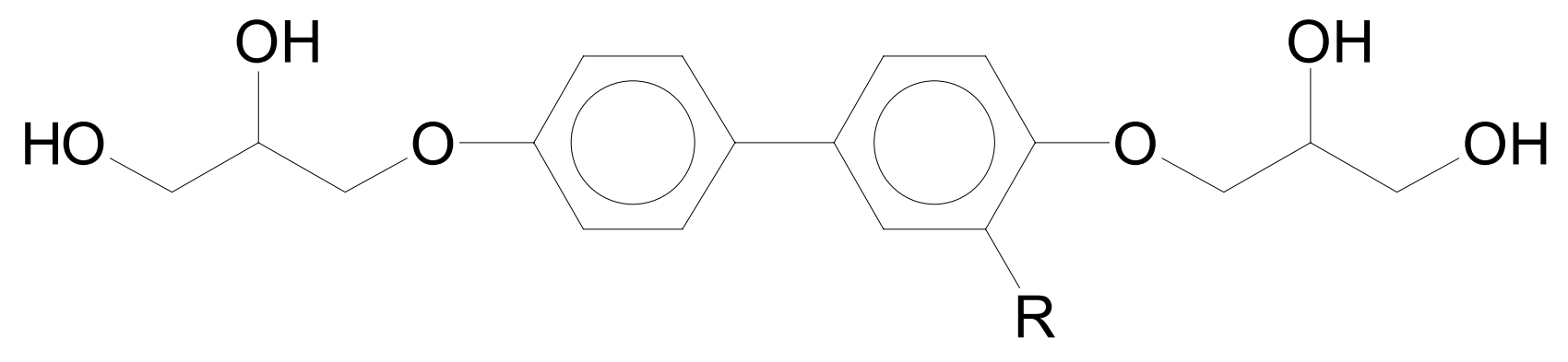

$\mathrm{R}=\mathrm{OCH}_{2} \mathrm{C}^{*} \mathrm{H}\left(\mathrm{CH}_{3}\right) \mathrm{O}\left(\mathrm{CH}_{2}\right)_{11} \mathrm{C}_{8} \mathrm{~F}_{17}$ 

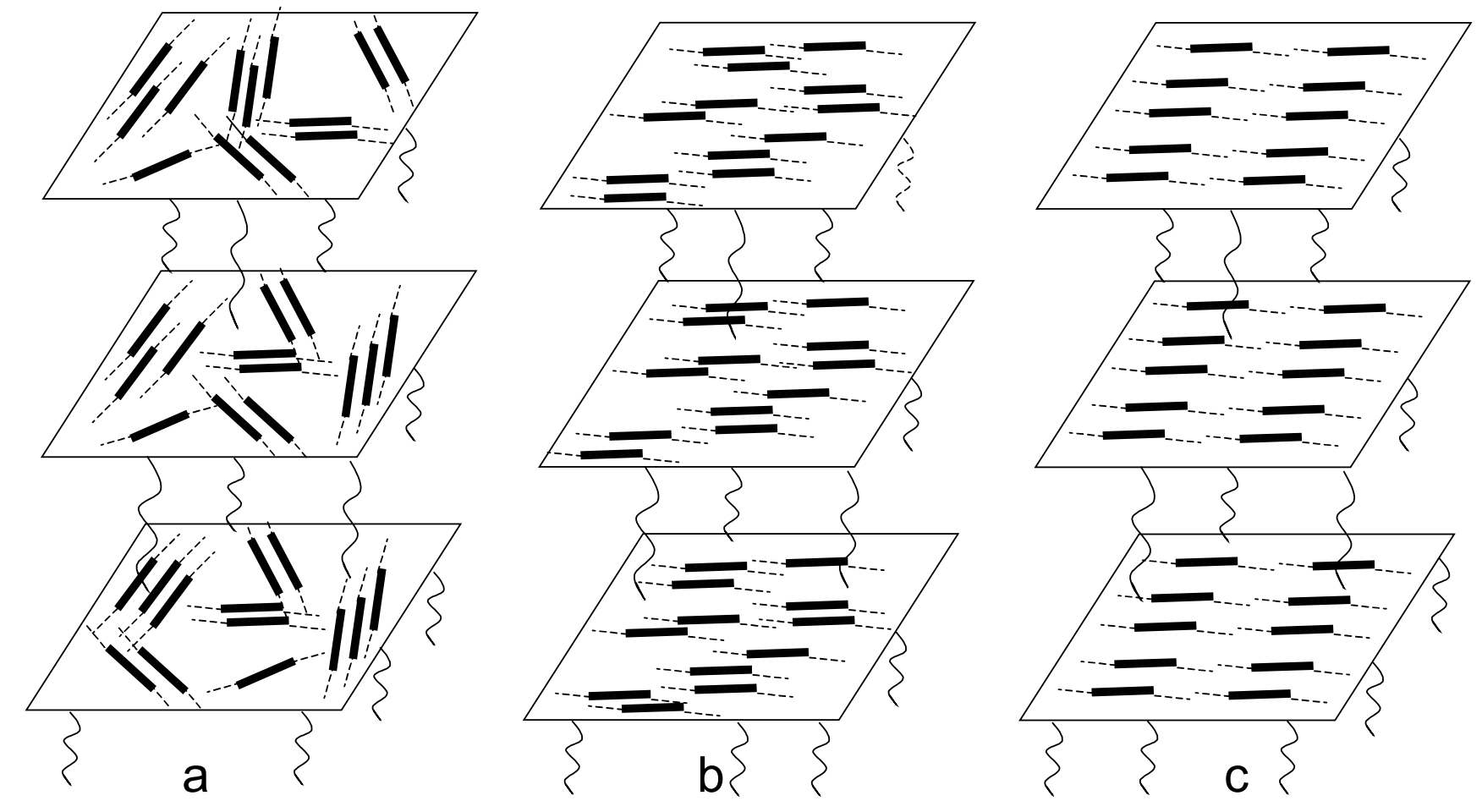


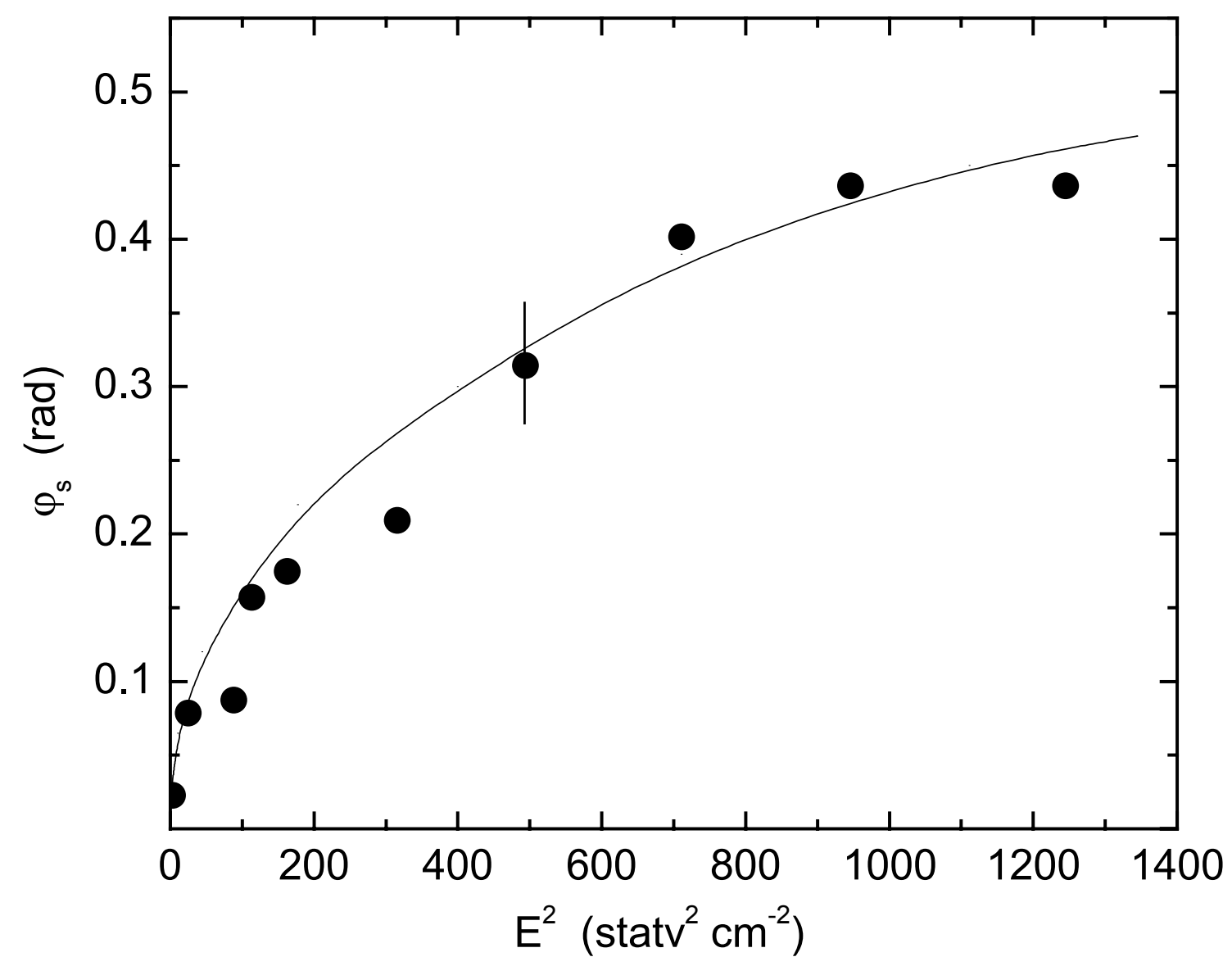




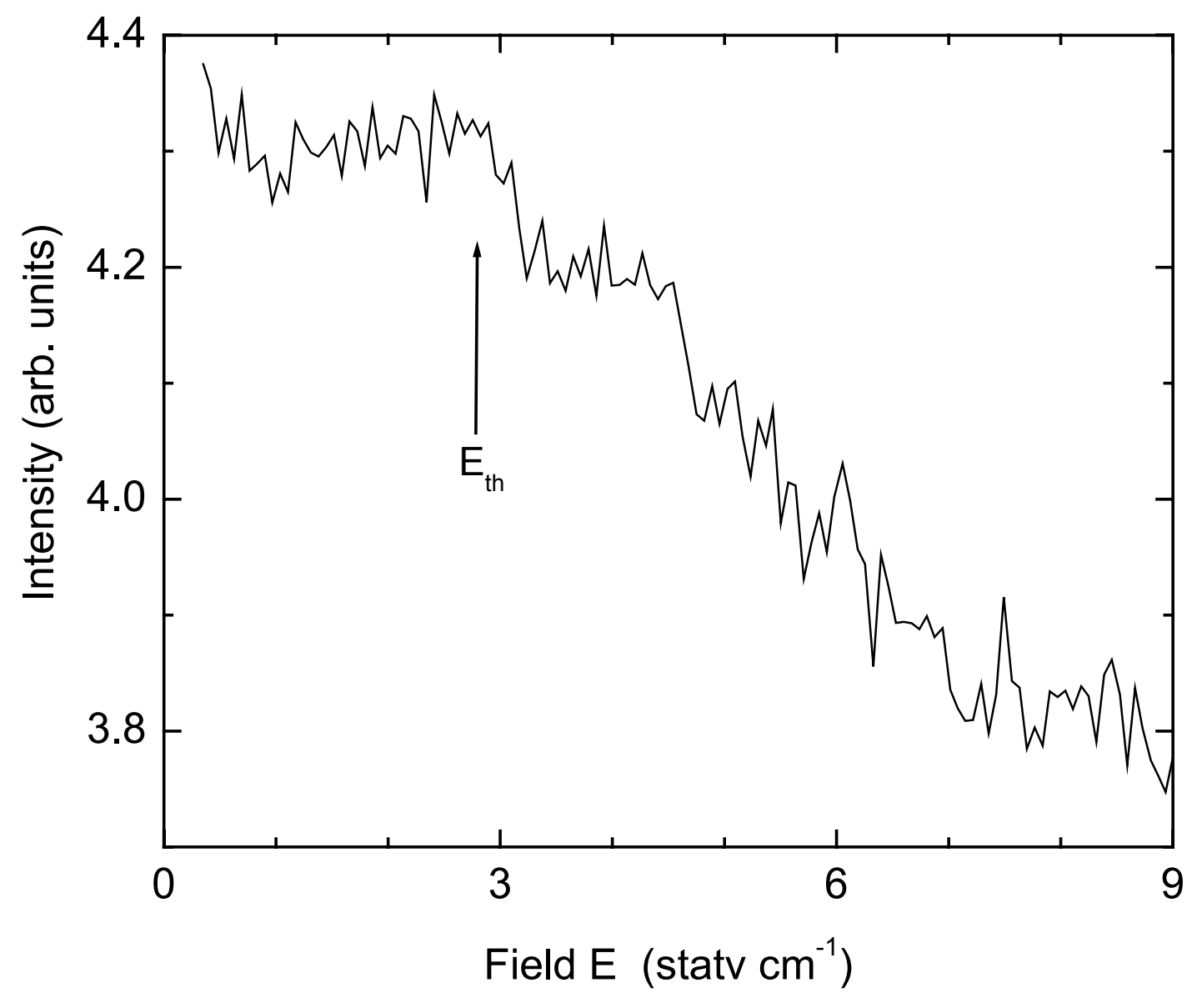

